\title{
Reviewers for Eye 1998
}

The Editor would like to thank the following, who have acted as reviewers for Eye in the past year:

Mr J. Acheson

Mr J.R. Ainsworth

Mr. W. Amoaku

Dr R.S. Amos

Mr A. Ansons

Mr W. Ayliffe

Mr C.S. Bailey

Mr S. Baker

Dr M. Barker

Mr K. Barton

Mr A.K. Bates

Mr M. Batterbury

Mr M.T. Benson

Mr P. Bloom

Mr D. Boase

Dr R. Bonshek

Mr J. Bradbury

Mr D. Broadway

Professor A.J. Bron

Mr D. Brosnahan

Mr J. Burke

Mr C.R. Canning

Mr A. Cassels-Brown

Mr A. Chandna

Dr K.S. Channer

Professor N. Charman

Dr B. Chauhan

Mr P.B. Chell

Mr H. Cheng

Mr P. Chew

Mr I. Chisholm

Ms A. Churchill

Mr C. Claoué

Ms K.G Claridge

Dr B.J. Clark

Mr D. Clark

Mr. L. Clearkin

Mr M. Cole

Mr S. Cook

Mr R. J. Cooling

Ms M.C. Corbett

Mr P.G.J. Corridan

Mr D. Cottrell

Dr S. Coupland

Dr I. Cree

Dr M.J. Cree
Mr I.A.C. Cunliffe

Dr Z. Currie

Mr J.K.G. Dart

Mr K. Davey

Dr J. Davis

Mr J. Devereux

Mr B. Dhillon

Mr A.D. Dick

Miss J. Dickinson

Professor P.N. Dilly

Mr P.M. Dodson

Mr R.M.L. Doran

Dr W. Douthwaite

Mr R. Downes

Mr J. Downie

Professor H.S. Dua

Professor G. Dutton

Miss E. Eagling

Professor D.L. Easty

Mr C. Edelsten

Mr M. Edwards

Mr N.M. Evans

Mr G. Fahy

Dr R. Feldman

Ms L. Ficker

Professor A.R. Fielder

Mr F. Figueiredo

Dr A.R.W. Forrest

Mr A. Foss

Dr A. Foster

Mr Paul Foster

Miss W. Franks

Mr D.G. Frazer

Mrs M. Freeman

Dr J. Frucht-Perry

Dr P.J. Gallagher

Mr N. Galloway

Mr E. Garway-Heath

Mr D. Gawkroger

Dr C. Geijssen

Dr P. Gerrish

Mr A. Gibson

Mr J. Gibson

Mrs C. Gilbert
Dr D.C. Gleeson

Dr J. Goepel

Dr D.G. Goodhead

Dr E.M. Graham

Dr S. Green

Ms H. Griffiths

Dr I. Haefliger

Mr P. Hamilton

Mr S. Harding

Ms C. Harper

Miss P.M. Hart

Mr J. Haslam

Dr E. Higginbotham

Dr J.C. Hill

Professor D.W. Hill

Mr J. Hillman

Dr P. Hiscott

Professor G. Holder

Dr F. Holz

Miss M. Hope-Ross

Dr S. Howell

Dr C. Hudson

Mr J. Hungerford

Mr P.G. Hykin

Mr D. Inglesby

Dr J. W. Ironside

Dr P. Jackson

Mr T. James

Dr Henry Jampel

Professor G.J. Johnson

Mr N.A. Johnson

$\mathrm{Mr}$ N. Jones

Mr W.S.S. Karwatowski

Mr P. Keams

Dr J. Kempster

Ms S. Kennedy

Mr M. Kerr-Muir

Mr G.R. Kirkby

Professor C.M. Kirkness

Dr T. Kivelä

Professor T. Koustas

Dr T. Kriss

Mr P.M. Kyle 


Mr D.A. Laidlaw
Dr D.S.C. Lam
Mr D.F. Larkin
Mr M. Lavin
Dr D. Lee
Dr J. Lee
Mr J.P. Lee
Professor W.R. Lee
Dr M.S. Lennard
Mr I. Levy
Mr C. Liu
Mr C. Lloyd
Mr A.J. Lotery
Professor P.J. Luthert
Dr G.P.M. Luyten
Mr I.A. Mackie
Dr S. MacNeil
Miss R. Manners
Dr M. Markis
Mr I. Marsh
Mr R.J. Marsh
Mr P.J. McDonnell
Mr R.M. McFadzean
Mr J. McGalliard
Mr M. McGavin
Professor C.N.J. McGhee
Mr M.J. Menage
Dr A. Mermond
Dr J.M. Miller
Mr M. Miller
Dr Minassian
Ms S. Mitchell
Mr A.T. Moore
Dr C.M. Mooy
Mr J. Morgan
Mr S. Morgan
Mr B.J. Moriarty
Mr A.J. Morrell
Mr R.J. Morris
Dr I. Moseley
Mr I. Murdoch

\begin{tabular}{|c|c|}
\hline Mrs J. Noble & $\begin{array}{l}\text { Dr A.D. Singh } \\
\text { Dr K. Singh }\end{array}$ \\
\hline Mr C. O'Brien & Dr K. Sisley \\
\hline Mr D. O’Neill & Professor G. Skuta \\
\hline Professor N. Osborne & $\begin{array}{l}\text { Dr D.N. Slater } \\
\text { Mr J.J. Sloper }\end{array}$ \\
\hline Professor P. Palmberg & Dr M. Sloper \\
\hline Professor R.K. Parish & Mr. G.T.H. Smith \\
\hline Mr A. Parker & Mr J. Sparrow \\
\hline Mr C. Pavesio & Ms A. Spencer \\
\hline Mr C.O. Peckar & Mr S.R. Spencer \\
\hline Dr B. Piguet & Dr R. Sponsel \\
\hline Mr M. Potts & Mr M. Stanford \\
\hline Mr J. I. Prydal & $\begin{array}{l}\text { Mrs P. Stavrou } \\
\text { Mr L. Stevenson }\end{array}$ \\
\hline Dr G. Quaghebeur & Mr P. Sullivan \\
\hline Mr M. Quinn & $\begin{array}{l}\text { Dr R. Susanna } \\
\text { Dr B. Svedbergh }\end{array}$ \\
\hline Ms N.K. Ragge & \\
\hline Dr J. Rahi & Mr M.K. Tandon \\
\hline Professor L. Ramsey & Dr A. Tanna \\
\hline Dr R.C. Read & Dr K.D. Teichmann \\
\hline Dr M. Restori & Mr V.T. Thaller \\
\hline Mr T. Reuser & Mr A.B. Tullo \\
\hline Mr J. Richardson & \\
\hline Mr R.P.S. Richardson & Dr G.S. Venables \\
\hline Mr A.E.A. Ridgway & Mr S.A. Vernon \\
\hline Dr E. Ridgway & \\
\hline Ms L. Ridgway & Dr G. Wallace \\
\hline Mr G. Rose & Mr A. Watts \\
\hline Mr E.S. Rosen & Mr M. Watts \\
\hline Mr P. Rosen & Dr M. Wax \\
\hline Dr J.A. Royds & Mr R.A. Welham \\
\hline Miss I.M. Russell-Eggitt & $\begin{array}{l}\text { Professor R.O. Weller } \\
\text { Mr J. Whittle }\end{array}$ \\
\hline Mr J. Salmon & Dr J.M. Wild \\
\hline Mr J. Sandford-Smirh & Professor H. Williams \\
\hline Mr N. Sarkies & Mr S. Winder \\
\hline Dr D.V. Seal & Dr D. Winfield \\
\hline Ms H. Seward & Mr J. Winfield \\
\hline Dr U. Schlötzer-Schrehardt & Mr P. Wishart \\
\hline Mr P. Shah & Mr D. Wong \\
\hline Mr J. Sharkey & Mr C. Wood \\
\hline Mr G.A. Shun-Shin & Dr M. Woodhouse \\
\hline Miss G. Silvestri & Mr G. Woodruff \\
\hline
\end{tabular}

